\title{
Editorial on Special Issue "Cosmetic Safety: Ingredients, Type of Reactions Undesirable Effects, Cosmetovigilance"
}

\author{
Immacolata Caputo and Lidia Sautebin * \\ Department of Pharmacy, School of Medicine, University of Naples Federico II, Via Montesano 49, \\ 80131 Naples, Italy; immacolata.caputo@unina.it \\ * Correspondence: sautebin@unina.it; Tel.: +39-081678427
}

Academic Editor: Enzo Berardesca

Received: 7 February 2017; Accepted: 7 February 2017; Published: 8 February 2017

Regulation (EC) No 1223/2009 on cosmetic products ("Cosmetics Regulation") [1] is the existing regulatory framework for finished cosmetic products placed on the European Union (EU) market. According to Regulation (EC) No 1223/2009, "cosmetic products should be safe under normal or reasonably foreseeable conditions of use. In particular, a risk-benefit reasoning should not justify a risk to human health". The responsibility for ensuring consumer safety rests with the manufacturer that places the product on the market.

Although cosmetics on the EU market have high standards of safety and quality, undesirable effects (UEs) can occur although mainly consisting in mild to moderate and reversible cutaneous reactions (e.g., itching, redness, skin swelling or irritation, allergic reactions). According to Cosmetics Regulation, an UE means "an adverse reaction for human health attributable to the normal or reasonably foreseeable use of a cosmetic product" (Article 2,o) [1] whereas a serious undesirable effect (SUE) means "an undesirable effect which results in temporary or permanent functional incapacity, disability, hospitalization, congenital anomalies or an immediate vital risk or death" (Article 2,p) [1].

In order to ensure a high level of consumer's health protection Cosmetics Regulation established the Cosmetovigilance system (Article 23) [1]. The goal was to set up a system enabling, by the collection, evaluation and monitoring of SUEs, the identification of potential substances with negative effects on human health. A collaboration of all stakeholders (responsible persons, distributors, doctors, pharmacists, authorities and Member States) is needed to collect and analyse all SUE cases, although only responsible persons and distributors have the legal obligation to report SUEs to the competent authorities of the Member States. In this regard, the management of UEs and reporting of SUEs, in the EU, by Cosmetics Europe, which represents the European cosmetics industry, are presented in this Issue as well as the experiences and statistical evaluation of SUEs notified to the EU Member States. The high amount of SUEs related to hair dyes notified in the EU highlights the problem of sensitization to p-phenylendiamine which is well described in a case report describing sensitization caused by temporary tattoos containing PPD and the use of hair dyes. Another essential requirement to assure cosmetic safety is the availability of specific and sensitive analytical methods. In this Issue the evaluation of packaging, another important aspect considered in the Regulation (EC) No 1223/2009, as well as the detection in cosmetics of pharmacologically active substances, such as the case of some prostaglandins analogues, contained in eyelash enhancers, are reported. Regrettably, the challenge for the EU authorities is not only to detect the possible risk related to complying products (see Cosmetovigilance) or to regulate new substances not yet listed in the Regulation Annexes (see prostaglandin analogues) but also to detect the presence, on the EU market, of dangerous cosmetics not complying with the existing legislation and posing at risk consumer's health. In this regards, in this Issue is reported an analysis of microbiologically contaminated and over-preserved cosmetic products present in the European Rapid Alert System (RAPEX) data base, from 2008 to 2014. 
Finally, since cosmetic safety is anywhere a main goal, an overview on the evaluation of cosmetic safety in Brazil, one of the greatest consumers of cosmetic products in the world, is reported.

In conclusion the aim of the Special Issue "Cosmetic safety: ingredients, type of reactions, undesirable effects, cosmetovigilance" was to present some issues related to cosmetic safety which is anyhow a complex problem.

\section{References}

1. EUR-Lex Access to European Union Law. Regulation (EC) No 1223/2009 of the European Parliament and of the Council of 30 November 2009 on Cosmetic Products. Available online: http:/ / eur-lex.europa.eu/ legal-content/EN/TXT/?uri=uriserv:OJ.L_.2009.342.01.0059.01.ENG\&toc=OJ:L:2009:342:TOC (accessed on 2 February 2017).

(C) 2017 by the authors; licensee MDPI, Basel, Switzerland. This article is an open access article distributed under the terms and conditions of the Creative Commons Attribution (CC BY) license (http://creativecommons.org/licenses/by/4.0/). 